\title{
Maturational Changes in Airway Remodeling after Chronic Exposure to Ovalbumin in Sensitized Guinea Pigs: Role of Cell Renewal of Airway Resident Cells
}

\author{
YASUSHI OHKI, KENICHI TOKUYAMA, AKIRA SATO, HIDEKO NISHIMURA, \\ MASAHIKO TABATA, KATUMI TOMIYOSHI, TOMIO INOUE, HIROKAZU ARAKAWA, \\ MASAHIKO KATO, HIROYUKI MOCHIZUKI, AND AKIHIRO MORIKAWA \\ Department of Pediatrics [Y.O., K.T., A.S., H.N., M.T., H.A., M.K., H.M., A.M.], Department of Nuclear \\ Medicine [K.T., T.I.], Gunma University School of Medicine, Maebashi, Japan
}

\begin{abstract}
We wanted to know whether airway remodeling caused by chronic exposures to antigen differed depending on the degree of maturation of animals. We sensitized guinea pigs at different stages of maturation: juvenile (approximately $200 \mathrm{~g}$ in body weight), adult (400 g), and old animals (800 g). Then, animals were repeatedly challenged with inhaled ovalbumin $(0.3 \%$ or $3 \%$ ) or vehicle twice a week for $6 \mathrm{wk}$. After the final challenge, the lungs were excised for the histologic evaluation of changes in the thickness of the inner wall area (Ti), the smooth muscle area (Tm), and the outer wall area (To) in noncartilaginous airway dimensions. To clarify whether or not the observed changes were due to renewal of airway cells, we stained the samples with labeled nucleotide 5'-bromo-2'-deoxyuridine (BrdU), which we injected repeatedly during the challenge periods. Chronic exposures to antigen induced airway wall thickening regardless of their stages of maturation. However, prominent areas of thickening differed between the three groups. Ti increased more remarkably in juvenile and adult animals than in old ones. By contrast, Tm significantly increased only in old animals. BrdU

staining revealed more renewal of epithelial cells in juvenile and adult animals than in old ones (juvenile $\geqq$ adult $>$ old), suggesting that increased renewal of epithelial cells contributed to the thickening of Ti in juvenile and adult animals. By contrast, only a slight increase in smooth muscle cell renewal was found even in old animals, indicating that an increase in Tm was due to factors such as hypertrophy. These results show that the development of antigen-induced airway remodeling is partly modified by the degree of maturation of animals in vivo. (Pediatr Res 52: 525-532, 2002)

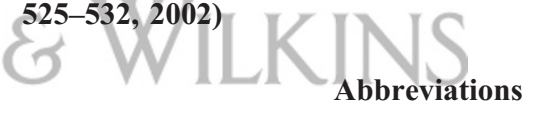

BrdU, 5'-bromo-2'-deoxyuridine

HSA, human serum albumin

OA, ovalbumin

$\mathbf{P i}$, internal perimeter

Ti, the thickness of the inner wall area

Tm, the thickness of smooth muscle

To, the thickness of the outer wall area
\end{abstract}

Recently, irreversible airflow limitation has been shown to be present in adults with asthma. This functional change is thought to be due to chronic allergic inflammation of the airways, and consequently, increased airway wall thickness that is caused by several mechanisms, including thickening of smooth muscles, subepithelial fibrosis, accumulation of inflammatory cells, and airway edema (1). These structural

Received November 5, 2001; accepted April 17, 2002.

Correspondence: Kenichi Tokuyama, M.D., Ph.D., Department of Pediatrics, Gunma University School of Medicine, Showa-machi 3-39-22, Maebashi, Gunma 371-8511, Japan; e-mail: tokuyama@showa.gunma-u.ac.jp

Supported, in part, by Health Sciences Research Grants (Research on Eye and Ear Sciences, Immunology, Allergy and Organ Transplantation) from the Ministry of Health, Labour and Welfare of Japan, and by the Grant-in-Aid for Scientific Research, (C) (2) No. 13670784, from the Ministry of Education, Science, Sports and Culture of Japan.

DOI: 10.1203/01.PDR.0000030882.20888.AF changes in the airways (i.e. airway remodeling) have been found in biopsy specimens of airways with a mild degree of severity in adult patients with asthma (2-4). However, it is not clear whether similar irreversible changes, which are seen in adults, could also occur in the airways of children with asthma. Longitudinal studies indicate that about $50 \%$ of all children with asthma are virtually free from symptoms within $10-20 \mathrm{y}$, although recurrences are sometimes seen in adulthood (5). Thus, it is possible that the development of allergic airway inflammation and, consequently, airway remodeling in asthma may show different features depending on age.

In animal models, we have shown that degrees of airflow obstruction and airway microvascular leakage caused by inflammatory mediators differ between immature and adult guinea pigs (6-10), suggesting that airway inflammatory re- 
sponses depend upon the stage of maturation. In other studies, maturational changes in the stimuli-induced airway smooth muscle responses have also been demonstrated in animal models, both in vivo (11) and in vitro $(12,13)$. These findings suggest that the degree of maturation of experimental animals may modify airway remodeling caused by chronic exposures to inflammatory stimuli.

In this study, we wanted to know whether the development of airway remodeling caused by chronic allergic inflammation differed depending on the degree of maturation of animals. Thus, we sensitized guinea pigs to OA at different stages of maturation. The animals were then repeatedly challenged with $\mathrm{OA}$, and the histologic changes of their airways were examined. To determine whether the structural changes were due to the renewal of airway resident cells (smooth muscle and epithelial cells) or other factors such as hypertrophy, nucleotide BrdU was also administered to the animals intraperitoneally (i.p.) as a marker of the cell renewal.

\section{MATERIALS AND METHODS}

Experimental animals. Experiments were performed using 72 pathogen-free male Dunkin-Hartley guinea pigs. The animals were divided into three groups depending on their stage of maturation at the time of the first sensitization: juvenile (209 \pm $10 \mathrm{~g}, 2$ wk after birth), adult (413 $\pm 11 \mathrm{~g}, 2$ mo after birth), and old animals (788 $\pm 39 \mathrm{~g}, 5$ mo after birth).

The animals of different age groups were further divided into three groups each: control animals $(n=4-6)$, and animals sensitized and repeatedly challenged with OA at concentrations of $0.3 \%(n=6-9)$ or $3 \%(n=6-9)$. As a control group, animals were only given Freund's complete adjuvant without $\mathrm{OA}$ and repeatedly challenged by normal saline, vehicle for OA. Some of the sensitized animals with different stages $(n=$ 5-7 for each group) were killed on d 21 after the first sensitization to obtain serum samples. The specific IgG1 antibody levels against $\mathrm{OA}$ in these sera were measured. To compare the amounts of intrapulmonary deposition of aerosolized $\mathrm{OA}$ in animals with different body weights, juvenile $(238 \pm 6 \mathrm{~g}, n=$ 4), adult (396 $\pm 9 \mathrm{~g}, n=4)$, and old animals $(738 \pm 19 \mathrm{~g}, n$ $=4)$ were administered aerosolized ${ }^{99 \mathrm{~m}} \mathrm{Tc}$-labeled HSA. All experiments in this study were approved by the institutional review board.

Sensitization and exposure protocol. Sensitization was performed according to the method described previously (14). In brief, $6 \mathrm{mg}$ of OA (albumin chicken egg, grade V, Sigma Chemical, St. Louis, MO, U.S.A.) was taken and emulsified in $1 \mathrm{~mL}$ Freund's complete adjuvant (Freund's complete adjuvant, Diatron, Tokyo, Japan) and injected intradermally at multiple sites $(12 \mathrm{mg} / \mathrm{kg})$ on $\mathrm{d} 0$ and 14 . The control group received vehicle for OA (only Freund's complete adjuvant) in the same manner. Chronic allergen challenge was started $1 \mathrm{wk}$ after the second sensitization. Allergen challenge was repeated twice a week for the first $5 \mathrm{wk}$ of the study and daily in the wk $6(15,16)$. Without any anesthesia, animals were fixed in the unit box $(11 \times 17 \times 21 \mathrm{~cm})$ with their heads protruding from the box and then into a nebulization box (unit style hypersensitization box for guinea pigs, Shizume Medical Inc., Tokyo,
Japan). The animals were challenged with aerosolized $0.3 \%$ or $3.0 \%$ OA solution or vehicle for OA ( $0.9 \%$ saline) produced by an ultrasonic nebulizer (NE-U12, Omron, Tokyo, Japan) for 10 min. The nebulization box allows four to 12 guinea pigs to breathe antigen (OA) simultaneously under an identical condition. A diffusion board inside the box equalizes the density of particles inside the box. All the animals sensitized and challenged with OA showed changes in respiratory pattern including tachypnea and cyanosis, whereas this was not the case in animals that inhaled vehicle for OA. In the case that an animal showed a severe change in breathing pattern (indrawing) and cyanosis before $10 \mathrm{~min}$ of exposure, the inhalation challenge at the time was withheld immediately after these symptoms appeared for ethical reasons. Diphenhydramine $(20 \mathrm{mg} / \mathrm{kg}$, Sigma Chemical) was given intraperitoneally $1 \mathrm{~h}$ prior to each challenge to prevent severe anaphylaxis. In all animals, $50 \mathrm{mg} / \mathrm{kg}$ of BrdU (Sigma Chemical) in $2 \mathrm{~mL}$ of distilled water was injected intraperitoneally twice weekly for the first $5 \mathrm{wk}$ of the study and daily in wk 6 according to the method by Wang et al. $(15,16)$.

Tissue preparation. Three days after the final challenge with OA, animals were killed with overdose of pentobarbital (500 $\mathrm{mg} / \mathrm{kg}$ i.p.). A cannula was introduced into the proximal portion of the trachea and the lungs were distended with 10-20 $\mathrm{mL}$ of $70 \%$ ethanol. The trachea was clamped until fixation was completed. Tissue specimens were cut into $6-\mu \mathrm{m}$-thick sections at mid-sagital slice, embedded in paraffin (17), and stained with hematoxylin and eosin (H-E). In selected animals, Luna staining was also performed to observe an infiltration of eosinophils in the airways.

The lungs were estimated to have been inflated to $50 \%$ of total lung capacity using values for the total lung capacity for guinea pigs calculated from a published algorithm (18).

Morphometric measurements. The image for each airway was incorporated into a personal computer (Presario 5201, Compaq, Houston, TX, U.S.A.) from a micrometer (IX-70, Olympus, Tokyo, Japan) through a charge-coupled device (CCD) camera and controller (M-3204C, Olympus, Tokyo, Japan). Airway dimensions were measured by the imageanalyzing software (NIH Image version 1.58, National Institutes of Health, Bethesda, MD, U.S.A.) and the digitizer (Pro-Draw, Seiko Instruments, Tokyo, Japan). The serial numbers of slides were blinded to the person who conducted the morphometric measurement until the measurement was completed. All cross-sectioned membranous airways with a shortto-long diameter ratio of $>0.6$ and that did not show bifurcation or disruption of the wall were analyzed. Pi, defined by the luminal surface of the epithelium, was traced as previously described and was used as a marker of airway size $(19,20)$. In addition, the perimeters at the basement membrane $(\mathrm{Pbm})$, outer border of smooth muscle $(\mathrm{Pm})$, and outer border of adventitia ( $\mathrm{Po}$ ) were traced. The areas defined by $\mathrm{Pi}, \mathrm{Pbm}, \mathrm{Pm}$, and Po were calculated (Ai, Abm, Am, Ao) as described previously (20). Then the thickness of each airway components for each airway was calculated (21).

The diameter of the maximally dilated airway was calculated and was referred to as the calculated luminal diameter: 


$$
\text { calculated diameter }=2 \times \text { radius }=\mathrm{Pi} / \pi
$$

The Ti was determined by subtracting the radius of a circle with a circumference equal to the internal perimeter from the radius of a circle with a circumference equal to the perimeter of the area internal to the basement membrane:

$$
\text { thickness of inner wall area }(\mathrm{Ti})=\sqrt{\mathrm{Abm}} / \pi-\sqrt{\mathrm{Ai}} / \pi
$$

The Tm and To were also calculated in the same manner from Ao, Am, and Abm. Total thickness of airway wall (Tt) was the sum of Ti, Tm, and To.

Number of tissue eosinophils. The number of eosinophils in the membranous airways was counted in all groups of animals with different ages. All cross-sectioned membranous airways with a short-to-long diameter ratio of $>0.6$ and that did not show bifurcation or disruption of the wall were analyzed. The number of eosinophils in each airway was quantified by dividing the raw number of these cells by $\mathrm{Pi}$ of each airway (counts $/ \mathrm{mm}$ ). The average of these values was calculated by measuring all the membranous airways countable, and was defined as the number of eosinophils of the individual value for each animal.

BrdU immunohistochemistry. Serial sections of the H-Estained slices were used for immunohistochemical detection of BrdU-immunoreactive nuclei. The sections were first dewaxed followed by incubating with $0.6 \% \mathrm{H}_{2} \mathrm{O}_{2}$ added $50 \%$ methanol solution to inhibit internal peroxidase activity. They were treated with acid $\left(1 \mathrm{~N}-\mathrm{HCl}, 37^{\circ} \mathrm{C}\right.$, for $\left.60 \mathrm{~min}\right)$ as a DNA denaturation step, and then digested with $0.01 \%$ trypsin (Sigma Chemical) for $10 \mathrm{~min}$ at $37^{\circ} \mathrm{C}$ to activate antigen. Then, they were incubated with 5\% normal goat serum (PCN5000, BioSource International, Carmarillo, CA, U.S.A.) in PBS as a blocking step to reduce nonspecific binding of secondary antibody. Mouse monoclonal anti-BrdU Ig (30268, BD Biosciences, San Jose, CA, U.S.A.) was then applied in PBS plus $5 \%$ fetal bovine serum for $1 \mathrm{~h}$. The slides were then washed three times in PBS for 5 min, and secondary antibody, biotylated goat anti-mouse Ig (DAKO, Glostrup, Denmark) was applied for $30 \mathrm{~min}$, followed by washing with PBS and incubation with peroxidase-conjugated streptavidine (DAKO) for $30 \mathrm{~min}$. Then, 3,3-diaminobenzidine tetrahydroride (DAKO) was added for 3-5 min to form colored reaction product. The sections were counterstained with Mayer's hematoxylin. BrdU point counting was assessed at $\times 400$ on both BrdU-stained sections and the H-E-stained sections used for airway wall dimension measurement.

To evaluate the degree of cell renewal in epithelial cells and smooth muscle cells, the "proliferation index" for each type of cells was arbitrary used by defining following formula: (numbers of BrdU-immunoreactive nuclei) $\div$ (numbers of nuclei shown by H-E staining $) \times 100(\%)$.

The epithelial cells were identified by their cuboidal or pseudostratified morphology and round nuclei. The smooth muscle cells represented spindle-shaped cells with spindleshaped nuclei. Any spindle-shaped nuclei separated by a gap or oriented nonparallel to the airway lumen was considered to represent a nonsmooth muscle cell and excluded from analysis.

Calculation of sublaryngeal deposition of aerosolized HSA. To compare the amount of tissue deposition of OA among animals with different body weights, another series of animals (immature, adult, and old animals, $n=4$ for each group) received $20 \mathrm{mCi}$ of nebulized ${ }^{99 \mathrm{~m}} \mathrm{Tc}-\mathrm{HSA}$ using the same apparatus and the same volume of solution used in chronic exposure procedure. After the completion of nebulization, the guinea pigs were killed by intraperitoneal overdose of urethane (Sigma Chemical, $5000 \mathrm{mg} / \mathrm{kg}$ i.p.) and succinylcholine (Yamanouchi Pharmaceuticals, Tokyo, Japan, $10 \mathrm{mg} / \mathrm{kg}$ i.p.). The trachea, main bronchus, and the lungs were excised, avoiding contamination by radionuclei deposited on the skin surface. The airways were separated into two parts (trachea and main bronchus, intrapulmonary airway, and the lung), then

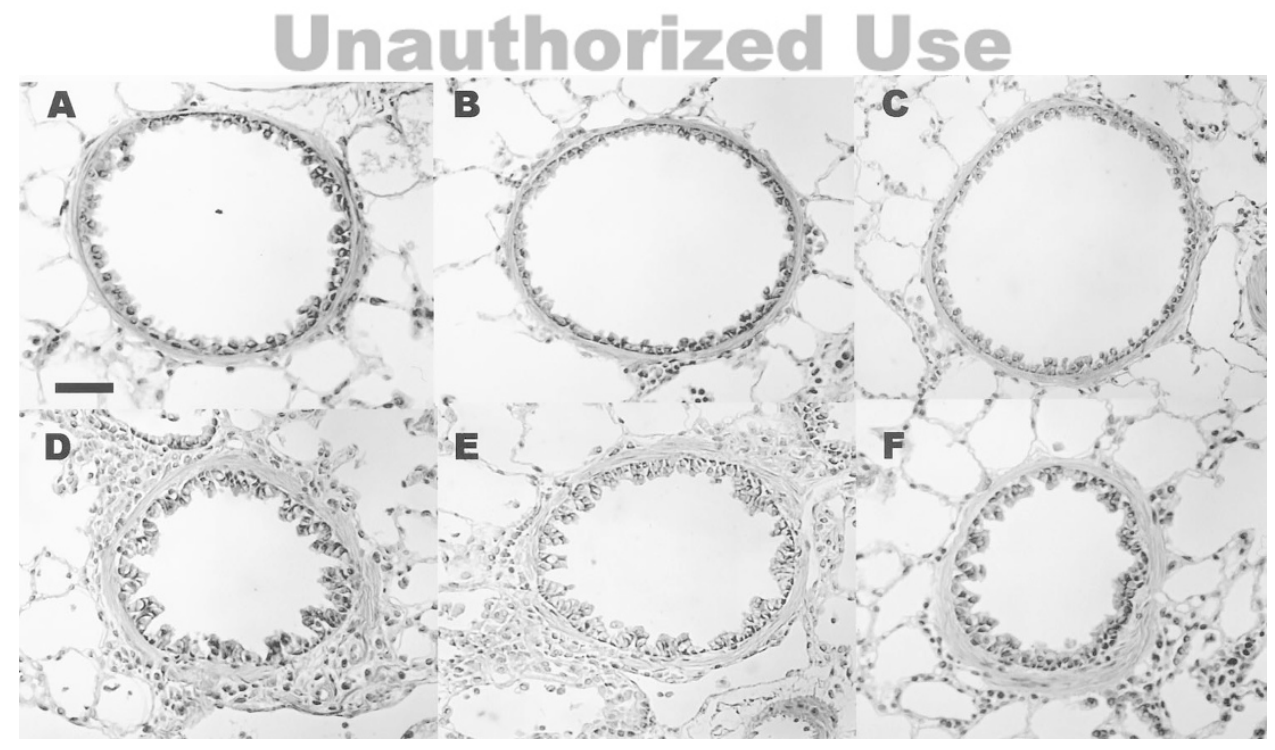

Figure 1. Representative photomicrographs of 6- $\mu \mathrm{m}$ sections of guinea pig smaller membranous airways (H-E staining). The sections of control animals ( $A$ : juvenile, $B$ : adult, $C$ : old animals) and those of animals sensitized and challenged with 3\% OA ( $D$ : juvenile, $E$ : adult, $F$ : old animals) are shown. In OA-challenged animals, the thickening of the inner and outer wall area was prominent in juvenile and adult animals $(D, E)$. In old animals, the thickening of the smooth muscle area was the predominant finding $(F)$. Size marker $=50 \mu \mathrm{m}$; magnification, $\times 400$. 


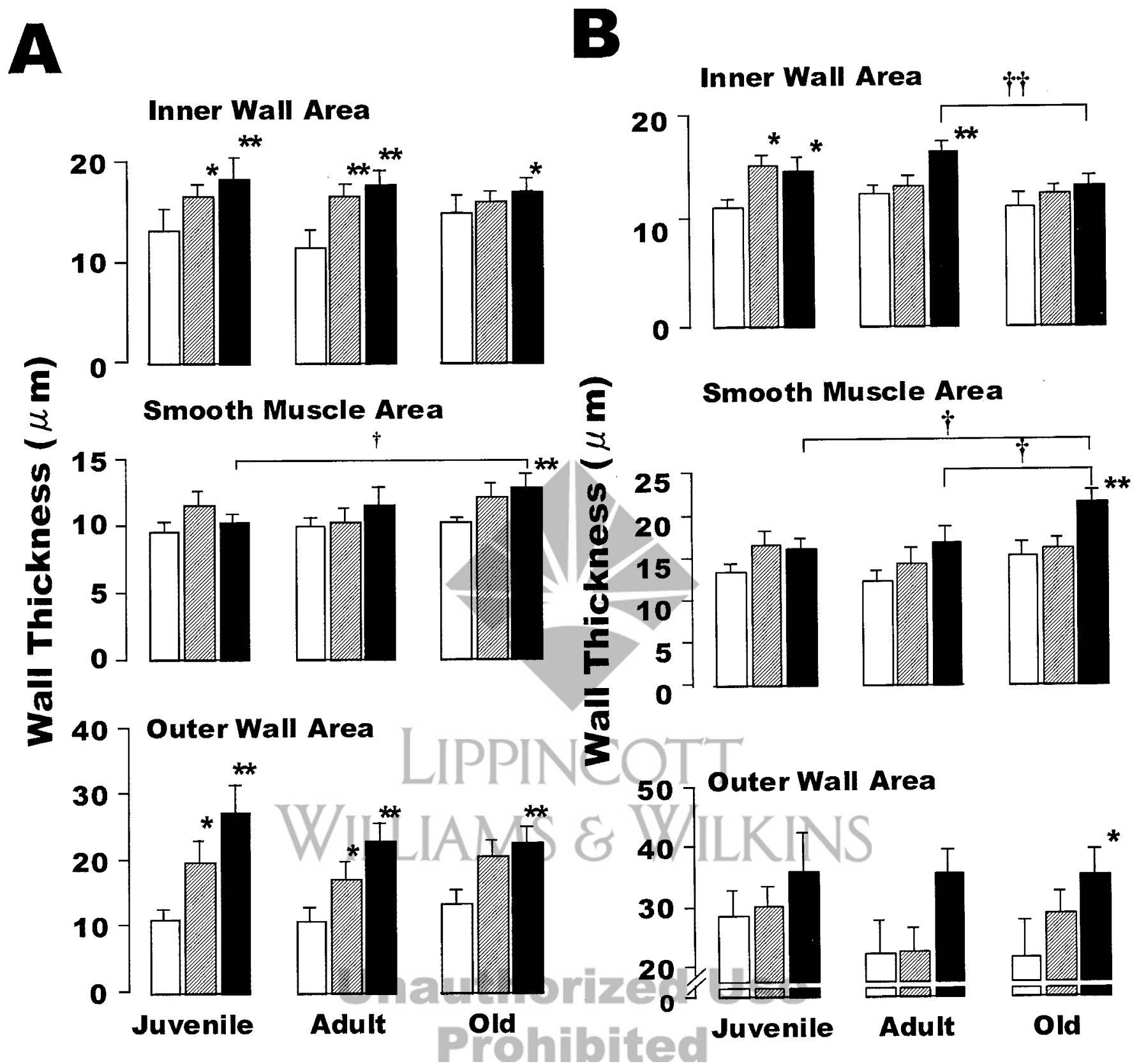

Figure 2. The effect of repeated exposure to OA on the thickness of airway wall components in smaller membranous airways $(A)$ and larger membranous airways $(B)$. The open column indicates the value of control animals. The hatched and closed columns indicate the values of animals sensitized and challenged with $0.3 \%$ and $3 \% \mathrm{OA}$, respectively. Values are mean \pm SEM. ${ }^{*} p<0.05$ and $* * p<0.01 v$ s control animals; $\uparrow p<0.05$ and $\dagger \dagger p<0.01$ between corresponding animals.

counted in a gamma counter (ARC-1000M, Aloka Co., Tokyo, Japan) to calculate the fractional deposition of aerosolized HSA. The counts were corrected for decay and background.

Assessment of specific IgG $\boldsymbol{G}_{1}$ antibody to $\mathrm{OA}$. To assess the degree of sensitization against OA before chronic OA inhalation, we measured OA-specific $\mathrm{IgG}_{1}$ antibody by ELISA in selected animals with or without sensitization as previously described (22) with minor modification. Microtiter plates were coated with $5 \mu \mathrm{g} / \mathrm{mL} \mathrm{OA}$ in $0.05 \mathrm{M} \mathrm{Na}_{2} \mathrm{CO}_{3} / \mathrm{NaHCO}_{3}$ buffer, $\mathrm{pH} 9.6$, and incubated overnight at $4^{\circ} \mathrm{C}$. After five washes in PBS/Tween, $100 \mu \mathrm{L}$ of guinea pig serum was added to the plates at doubling dilutions that ranged from 1:200 to 1:409,600 and incubated overnight at $4^{\circ} \mathrm{C}$. After another five washes, the plates were incubated with $100 \mu \mathrm{L}$ of $(1: 5000)$ rabbit anti-guinea pig $\operatorname{IgG}_{1}(9-486$, Nordic Immunological Laboratories, Tilburg, The Netherlands), followed by a further five washes with $100 \mu \mathrm{L}$ of peroxidase-conjugated swine anti-rabbit IgG (p-217, DAKO), both incubations being for $2 \mathrm{~h}$ at $37^{\circ} \mathrm{C}$. After three final washes, o-phenylenediamene substrate in phosphate-citrate buffer with hydrogen peroxide was added $10 \mathrm{~min}$ before measurement. The reaction was stopped with $4 \mathrm{M}$ sulfuric acid and the plate was read at an OD of 490 $\mathrm{nm}$. $\mathrm{IgG}_{1}$ titers were calculated by interpolation on a dilution curve, with doubling serum dilutions on the $x$ axis and ELISA reading at $490 \mathrm{~nm}$ on the $y$ axis. An ELISA reading above 0.10 at a dilution of 1:200 or more (above the 95\% confidence 


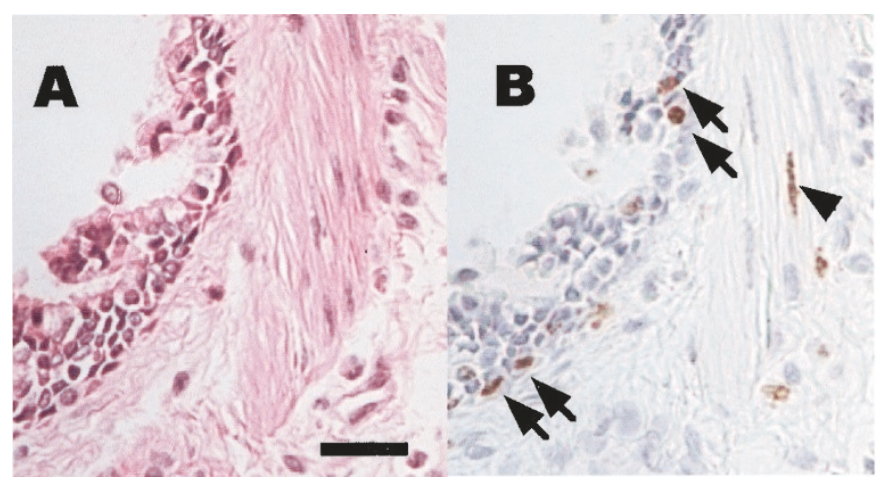

Figure 3. Representative photomicrographs of a membranous airway stained with H-E $(A)$ and BrdU immunohistochemistry $(B)$. Serial sections of the $\mathrm{H}$-E-stained slices were used for immunohistochemical detection of BrdUimmunoreactive nuclei. BrdU-immunoreactive nuclei of epithelial cells ( $a r-$ rows) and smooth muscle cell (arrow head) were stained in brown $(B)$. Size marker $=25 \mu \mathrm{m}$; magnification $\times 400$.

interval for control animals at a dilution of 1:200) was considered to be positive.

Data analysis. All data were expressed as mean \pm SEM unless otherwise mentioned. Nonparametric analysis of variance (Kruskal-Wallis test for unmatched pairs) was used to determine the significance of variance between groups. If a significance of variance was found, a Mann-Whitney $U$ test was performed to assess the significance of differences between groups. A $p$ value of $<0.05$ was considered to indicate statistical significance. Statistical analysis was performed using Statview version 4.1 (Abacus Concepts, Berkeley, CA, U.S.A.).

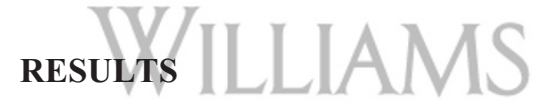

Growth of animals during the study period. Among 72 guinea pigs used in this study, two animals exposed to $0.3 \%$ and three animals exposed to $3.0 \%$ OA died because of anaphylactic shock during challenges. The remaining 67 animals were evaluated for the histologic examinations. At initial sensitization, the body weights of juvenile, adult, and old animals of control groups were $190 \pm 28,400 \pm 22$, and $857^{ \pm} \pm 69 \mathrm{~g}$, respectively. Those in animals sensitized and challenged with $0.3 \% \mathrm{OA}$ were $237 \pm 13,456 \pm 17$, and $816 \pm 46 \mathrm{~g}$, and in $3 \%$ OA-exposed animals were $197 \pm 13,379 \pm 6$, and $753 \pm$ $33 \mathrm{~g}$, respectively. At completion of the study, there was a tendency for the animals challenged with OA to show less weight gain compared with control animals, although this difference was not statistically significant in all age groups.

Morphometric measurements. Sixty-seven sections of lung tissue that contained 352 membranous bronchioles (nonalveolated, noncartilagenous airways) were examined. Based on the luminal diameter, membranous airways were divided into two categories: larger membranous airways $(400-700 \mu \mathrm{m}, n=$ $171)$ and smaller membranous airways $(<400 \mu \mathrm{m}, n=181)$. There was no significant difference in Pi among all groups of animals studied, indicating that similar-sized airways were examined (data not shown). In smaller membranous airways, $\mathrm{Ti}$ and To significantly increased after OA challenges in a dose-dependent manner in all age groups (Figs. 1 and $2 A$ ). Tm in old animals significantly increased after 3\% OA exposure, whereas this was not seen in juvenile and adult animals (Figs. 1 and $2 A$ ). In larger membranous airways, To showed a dose-dependent increase in all groups. However, the increase in Tm was only significant in old animals, whereas that in $\mathrm{Ti}$ was so only in juvenile and adult ones (Fig. 2B).

Number of tissue eosinophils. As shown in Table 1, the number of tissue eosinophils increased dose dependently in animals sensitized and challenged with OA. A significant increase was observed in animals given 3\% OA compared with corresponding animals given vehicle in all age groups.

Proliferation indices assessed by BrdU point counting. A representative photomicrograph showing BrdU-positive cells is shown in Figure 3. In both smaller (Fig. $4 A$ ) and larger membranous airways (Fig. 4B), the proliferation indices of the epithelial cells increased in a dose-dependent manner except for the larger airways in old animals. The proliferation indices of the epithelial cells in juvenile animals that inhaled 3\% OA was 3- to 4-fold higher than those of controls of the corresponding age, and was significantly higher than that in the old animals that inhaled $3 \% \mathrm{OA}$. By contrast, increases in the proliferation indices of the smooth muscle cells were not remarkable and did not differ among different age groups.

Calculation of sublaryngeal deposition of aerosolized HSA. Quantification of aerosolized HSA revealed that 0.0097 $\pm 0.0010 \%, 0.0036 \pm 0.0015 \%$, and $0.0062 \pm 0.026 \%$ of the initial nebulized doses were deposited in trachea and main bronchi in juvenile, adult, and old animals, respectively. In the intrapulmonary airways and the lung, these proportions were $0.023 \pm 0.004 \%, 0.040 \pm 0.010 \%$, and $0.036 \pm 0.004 \%$ in these animâls, respectively. The amounts of deposition in trachea and main bronchi were significantly higher in old animals than in juvenile and adult ones $(p<0.05)$. However, there was no significant difference among the three groups in the deposited proportion in intrapulmonary airways and the lung (Fig. 5).

Specific $\operatorname{Ig} G_{1}$ antibodies. Specific $\operatorname{IgG}_{1}$ antibody levels against $O A$ in each animal are shown in Figure 6. The OAspecific $\operatorname{IgG}_{1}$ antibody levels significantly elevated in sensitized groups in juvenile, adult, and old animals compared with control adult animals. There was no significant difference in the value of titers among groups.

Table 1. Number of eosinophils in the membranous airways after repeated ovalbumin exposure

\begin{tabular}{lccc}
\hline \multirow{2}{*}{$\begin{array}{c}\text { Treatment } \\
\text { group }\end{array}$} & \multicolumn{2}{c}{ Number of eosinophils (cells/mm internal perimeter) } \\
\cline { 2 - 4 } & Juvenile & Adult & Old \\
\hline Saline & $7.1 \pm 1.5(6)$ & $9.5 \pm 1.6(6)$ & $15.7 \pm 3.8^{*}(6)$ \\
$0.3 \%$-OA & $21.5 \pm 7.7(6)$ & $22.5 \pm 7.0(6)$ & $34.0 \pm 11.1(6)$ \\
$3 \%$-OA & $48.3 \pm 8.8 \dagger(6)$ & $49.3 \pm 11.6 \dagger(6)$ & $73.7 \pm 14.4+(6)$ \\
\hline
\end{tabular}

Eosinophil counts in the membranous airways were compared between animals sensitized and challenges with $\mathrm{OA}$ and control animals (saline). Values were eosinophil numbers per internal perimeter of the airway (cells/ $\mathrm{mm}$ ), mean \pm SEM. Number in parenthesis is the number of animals utilized.

${ }^{*} p<0.05$ vs saline-exposed juvenile animals.

$\dagger$ and $\ddagger, p<0.05$ and $p<0.01$ compared to control animals. 
A

$\mathbf{B}$
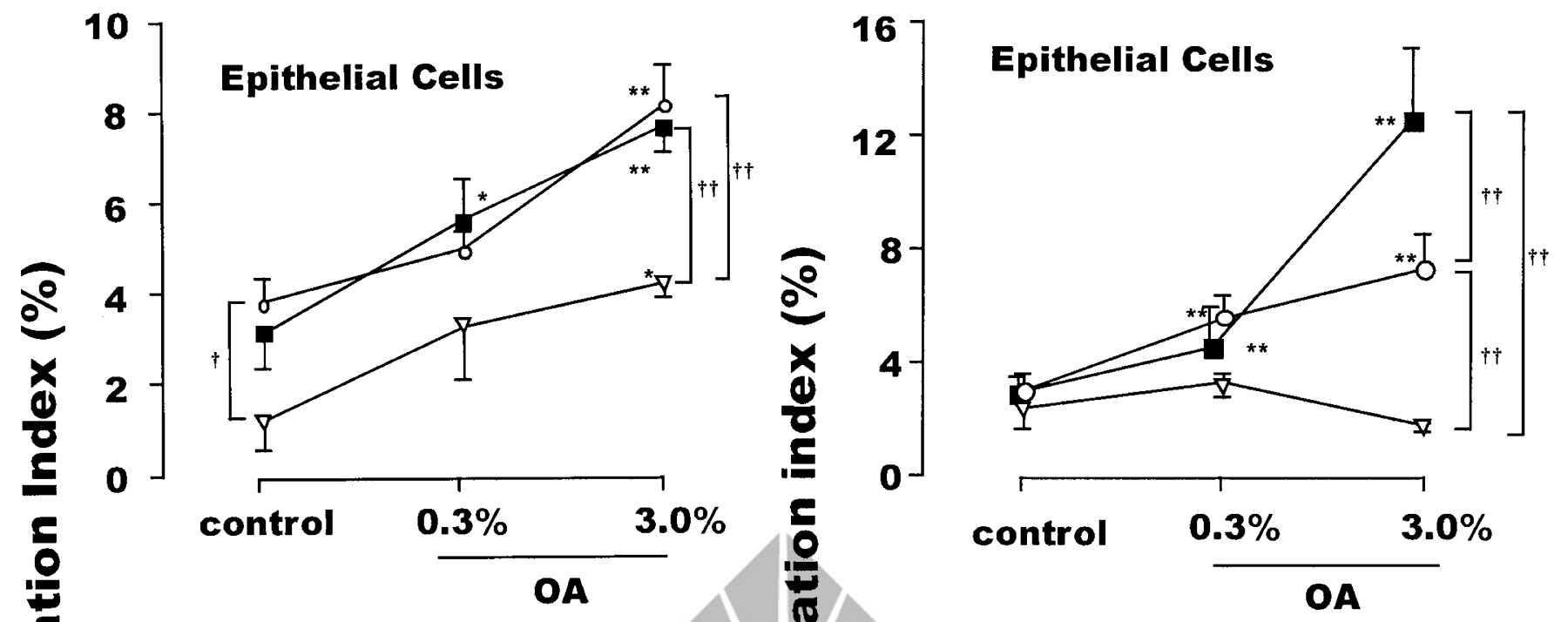

힌

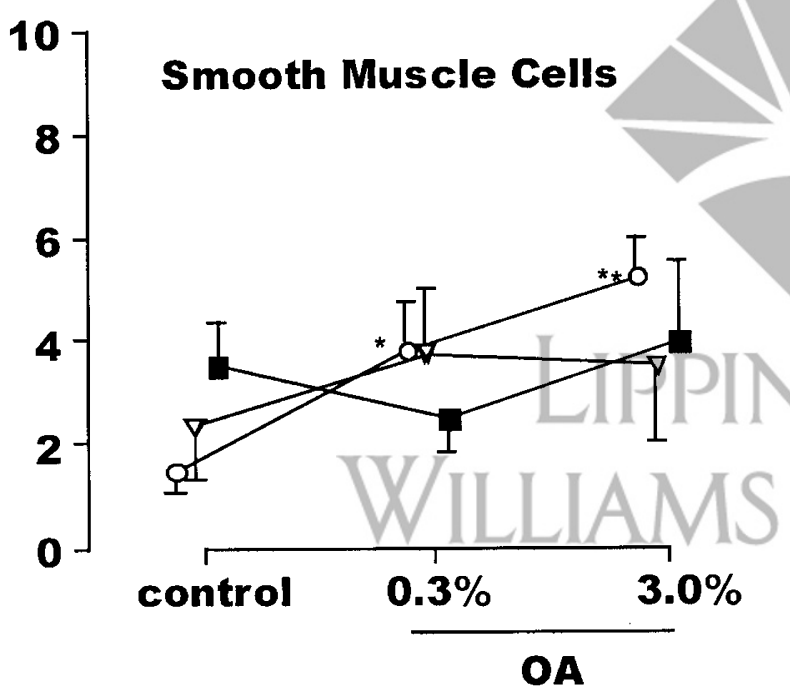

10

\section{Smooth Muscle Cells}

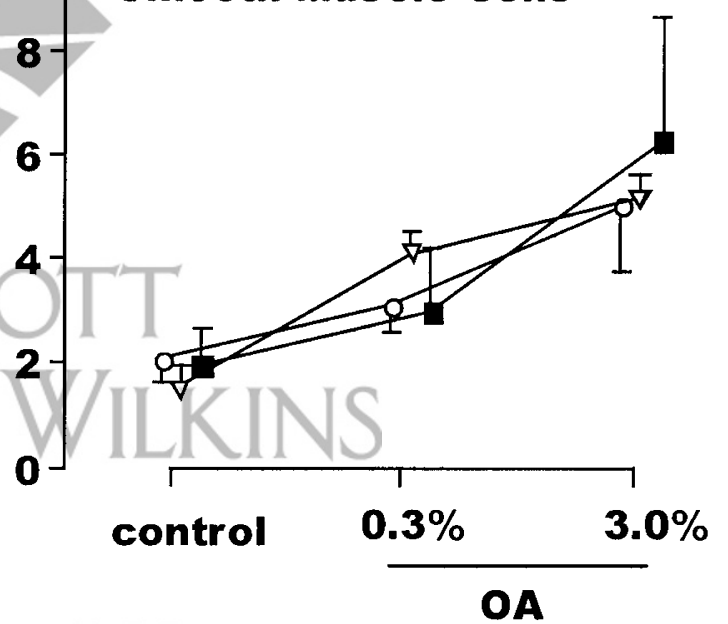

Figure 4. Proliferation indices for epithelial cells and smooth muscle cells in smaller $(A)$ and larger $(B)$ membranous airways. The closed square, open circle, and open triangle indicate the values of juvenile, adult, and old animals, respectively. Values are mean \pm SEM. ${ }^{*} p<0.05$ and $* * p<0.01 v s$ control animals; $\dagger p<0.05$ and $\dagger \dagger p<0.01$ between corresponding animals.

\section{DISCUSSION}

To determine whether there was any age-related differences in the development of airway remodeling after chronic exposures to antigen, we repeatedly exposed OA to three groups of guinea pigs at different stages of maturation, and compared the morphologic changes in their airways. As far as we know, this is the first study to investigate age-related differences in airway remodeling caused by chronic allergic inflammation. We have shown that chronic exposure to antigen induced airway wall thickening regardless of their stages of maturation. This structural change was suggested to be related to allergic airway inflammation, because the number of tissue eosinophils increased dose dependently in animals of all age groups. However, the details of thickening varied among the three groups. In juvenile and adult animals, a significant thickening was found at the inner wall, but not at the smooth muscle area. By contrast, it was seen at the smooth muscle area rather than at the inner wall area in the case of old animals. The outer wall area showed a dose-dependent thickening in all thee groups. BrdU staining revealed that epithelial cell renewal was significantly greater in juvenile and adult animals than in old ones (juvenile $\geqq$ adult $>$ old). By contrast, only a slight increase in the cell renewal was seen in the case of smooth muscle areas in all groups of animals without any age-related difference.

It was interesting that, in juvenile and adult animals, the prominent area of thickening was the inner wall, but not the smooth muscle area, although an inverse relationship was found in old ones. Age-related differences in the cell turnover of airway epithelial cells may have partly contributed to this difference. In rats, steady-state epithelial cell turnover has been shown to decrease as the age of the animals increased (23). It has also been shown that aging in animals affects on the airway 

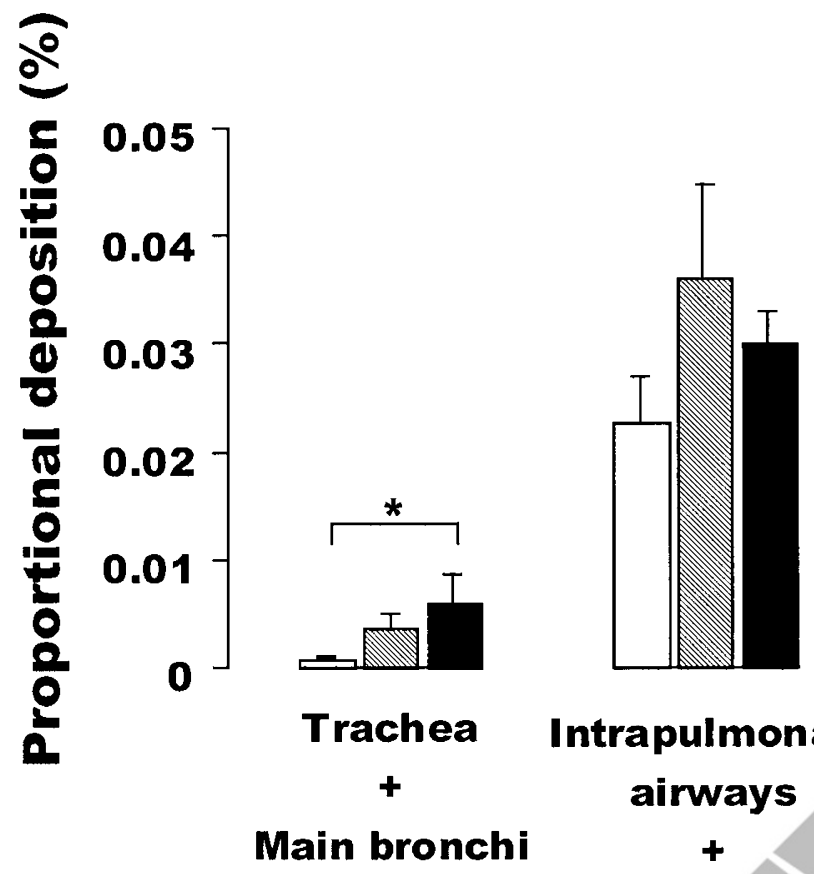

Intrapulmonary airways

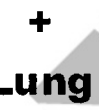

Figure 5. Sublaryngeal deposition of aerosolized ${ }^{99 \mathrm{~m}}$ Tc-labeled HSA in animals with different body weights. The airways were divided into two portions: the trachea and the main bronchi, and the intrapulmonary airways and the lung. The proportional deposition against total aerosolized radiolabeled HSA (\%) in each portion is shown. The open, hatched, and closed columns indicate the values of juvenile, adult, and old animals, respectively. Values are mean \pm SEM. ${ }^{*} p<0.05$ between groups.

epithelial proliferation response after various stimuli (24). For example, the proliferation index of the bronchiolar epithelial cells is demonstrated to be higher in 1-mo-old than in 25-moold rats after exposure to nitrogen dioxide (25). In the present study, the renewal of epithelial cells was significantly more in juvenile and adult animals than in old ones. Thus, it is possible that a greater degree of proliferation of the epithelial cells in the airways of younger animals induced, at least in part, more thickening of the inner wall area of these animals compared with that of old ones. It is not negligible that a difference in the degree of subepithelial fibrosis, another feature of airway remodeling (26), may have contributed to this age-related difference in the thickening of membranous airways. However, in the present study, it was impossible to evaluate the role of this structural change in the thickening of the inner wall areas, because the subepithelial layer was too thin to quantify this area. Further studies would be required to clarify this possibility.

In contrast to the inner wall area, the thickness of the smooth muscle area after chronic OA exposure significantly increased only in old animals, showing that the smooth muscle area is more prone to become thick in old animals than in juvenile or adult ones. A recent report by Bai et al. (27) suggests that similar tendency may occur in humans. They analyzed the airways of patients of various ages with fatal asthma and found that the duration of asthma and older age were associated with progressive remodeling of the airway wall, including a pro-

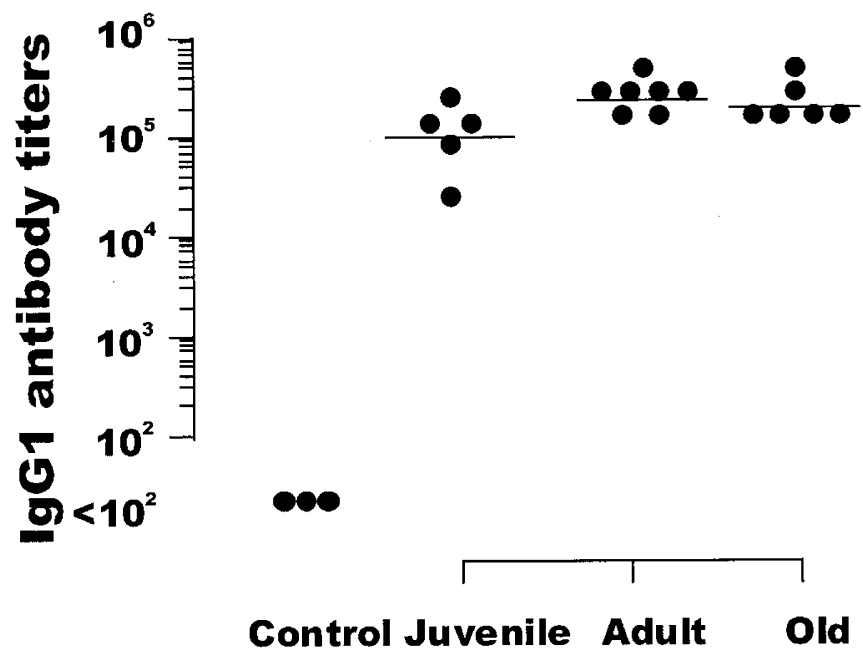

Figure 6. OA-specific $\operatorname{IgG}_{1}$ antibody titers. Sensitized animals of different age groups had significantly higher $\operatorname{IgG}_{1}$ titers than control adult animals. There was no age-related difference in the value of titers.

gressive increase in the airway smooth muscle volume and a greater reduction in the airway lumen. As for the mechanism of thickening of the smooth muscle area, it has been shown that smooth muscle cells replicate very poorly in vivo, although they proliferate actively in vitro (28). Various stimuli including growth factors, cytokines, contractile agonists, and mechanical stress are known to induce both smooth muscle hyperplasia and hypertrophy as chronic changes (29). Clinically, an increase in airway myocyte proliferation (hyperplasia) as well as hypertrophy has been reported in the airways of asthmatic patients (30). In the present study, only a slight increase in the smooth muscle cell renewal was found even in old animals, indicating that an increase in $\mathrm{Tm}$ in old ones was due not to hyperplasia, but to other factors such as hypertrophy. Further studies would be required to elucidate the exact mechanism of this agerelated difference.

Differences in the amounts of local deposition of OA among animals with different body weights may have caused the different features of airway remodeling in the present study. To address this possibility, we calculated the sublaryngeal deposition of ${ }^{99 \mathrm{~m}} \mathrm{Tc}-\mathrm{HSA}$ among the three age groups of animals. Our results showed that deposition in the trachea and main bronchi was significantly greater in old animals than in juvenile ones. However, there was no significant difference among the three groups in the proportions deposited in the intrapulmonary airways and the lungs. Thus, it is unlikely that different amounts of deposited antigen have caused the different structural changes seen in the peripheral airways.

It is possible that sensitization and challenges with $\mathrm{OA}$ induced different immunologic responses among groups, causing different structural changes in the airways. An increased production of locally produced cytokines and growth factors from inflammatory cells such as mast cells together with the Th2 environment leads to activation of myofibroblasts, excessive matrix deposition, and production of mediators that propagate and amplify the remodeling responses throughout the airway wall (26). Thus, we examined $\mathrm{OA}$-specific $\operatorname{IgG}_{1}$ antibody levels in each group of animals to assess antibody 
production capability. We found no difference in OA-specific antibody levels among the three age groups. However, it is not completely negligible that the difference in structural changes among groups has resulted from that in systemic and local immunologic responses.

Our present results show that airway remodeling caused by chronic allergic inflammation may be partly modified by the stage of maturation in guinea pigs. If this is the case in humans, the development of airway remodeling in asthma may be, to some extent, different between children and adults, especially the elderly.

Acknowledgments. The authors thank Mrs. Tomoko Endo and Chinori Iijima for their technical assistance and for caring for the animals. We also thank Dr. Hirokazu Kimura, Gunma Prefectural Institute of Public Health and Environmental Science, Maebashi, for his technical assistance.

\section{REFERENCES}

1. National Institutes of Health 1995 Global Initiative for Asthma. NIH Publication No 95-3659. National Institutes of Health, Bethesda, MD, pp 1-8

2. Vignola AM, Chanez P, Campbell AM, Souques F, Lebel B, Enander I, Bousquet J 1998 Airway inflammation in mild intermittent and in persistent asthma. Am J. Respir Crit Care Med 157:403-409

3. Carroll N, Elliot J, Morton A, James A 1993 The structure of large and small airways in nonfatal and fatal asthma. Am Rev Respir Dis 147:405-410

4. Jeffery PK, Wardlaw AJ. Nelson FC, Collins JV, Kay AB 1989 Bronchial biopsies in asthma: an ultrastructural, quantitative study and correlation with hyperreactivity. Am Rev Respir Dis 140:1745-1753

5. Sly RM 2000 Nelson Textbook of Pediatrics, 16th Ed. WB Saunders, Philadelphia, pp $664-680$

6. Arakawa H, Tokuyama K, Yokoyama T, Mochizuki H, Morikawa A, Kuroume T, Barnes PJ 1992 Effect of maturation on histamine-induced airflow obstruction and airway microvascular leakage in guinea pig airways. Eur J Pharmacol 215:51-56

7. Tokuyama K, Yokoyama T, Morikawa A, Mochizuki H, Kuroume T, Barnes PJ 1993 Attenuation of tachykinin-induced airflow obstruction and microvascular leakage in immature airways. Br J Pharmacol 108:23-29

8. Yokoyama T, Tokuyama K, Morikawa A, Kuroume T 1993 Bradykinin is less potent in causing airway microvascular leakage in immature than in adult guinea-pigs. Role of neutral endopeptidase. Eur J Pharmacol 236:427-432

9. Arakawa H, Lötvall J, Kawikova I, Tokuyama K, Löfdahl C, Skoogh B 1994 Effect of maturation on airway plasma exudation induced by eicosanoids in guinea pigs. Eur J Pharmacol 259:251-257

10. Arakawa H, Tokuyama K, Mochizuki H, Morikawa A, Lötvall J 1997 Effect of maturation on allergen-induced airflow obstruction and airway plasma exudation in sensitized guinea pigs. Int Arch Allergy Immnol 112:196-202
11. Mhanna MJ, Dreshaj IA, Haxhiu MA, Martin RJ 1999 Mechanism for substance $\mathrm{P}$-induced relaxation of precontracted airway smooth muscle during development. Am J Physiol 276:L51-L56

12. Rhoden KJ, Souhrada M, Douglas JS 1997 Maturational changes in Na (+)-K+ pump activity in guinea pig airway smooth muscle. Exp Lung Res 23:333-345

13. Tepper RS, Du T, Styhler A, Ludwig M, Martin JG 1995 Increased maximal pulmonary response to methacholine and airway smooth muscle in immature compared with mature rabbits. Am J Respir Crit Care Med 151:836-840

14. Mirza ZN, Tokuyama K, Arakawa H, Kato M, Mochizuki H, Morikawa A 1998 Inhaled procaterol inhibits histamine-induced airflow obstruction and microvascular leakage in guinea-pigs airways with allergic inflammation. Clin Exp Allergy 28:644652

15. Wang ZL, Bramley AM, Pare PD, Bai TR 1994 Chronic fenoterol exposure increases in vivo and in vitro airway responses in guinea pigs. Am J Respir Crit Care Med 149:960-965

16. Wang ZL, Walker BAM, Weir TD, Yarema MC, Roberts CR, Okazawa M, Pare PD, Bai TR 1995 Effect of chronic antigen and $\beta 2$ agonist exposure on airway remodeling in guinea pigs. Am J Respir Crit Care Med 152:2097-2104

17. Tokuyama K, Kuo HP, Rohde JA, Barnes PJ, Rogers DF 1990 Neural control of goblet cell secretion in guinea pig airways. Am J Physiol 270:L108-L115

18. Pare PD, Michoud MC, Boucher RC, Hogg JC 1979 Pulmonary effects of acute and chronic antigen exposure of immunized guinea pigs. J Appl Physiol 46:346-353

19. James AL, Hogg JC, Dunn LA, Pare PD 1988 The use of the internal perimeter to compare airway size and to calculate smooth muscle shortening. Am Rev Respir Dis 138:136-139

20. Bai TR, Eidelman DH, Hogg JC, James AL, R. Lambert RK, Ludwig S, Martin J, McDonald DM, Mitzner WA, Okazawa M, Pack RJ, Pare PD, Schellenberg RR, Tiddens HA, Wagner EM, Yager D 1994 Proposed nomenclature for quantifying subdivisions of the airway wall. J Appl Physiol 77:1011-1014

21. Mochizuki H, Ohki Y, Arakawa H, Tokuyama K, Morikawa A 1999 Effect of ultrasonically nebulized distilled water on airway epithelial cell swelling in guinea pigs. J Appl Physiol 86:1505-1512

22. Arakawa H, Lötvall J, Kawikova I, Tee R, Hayes J, Löfdahl CG, Newman Taylor AJ, Skoogh BE 1993 Airway allergy to trimellitic anhydride in guinea pigs: different time courses of IgG1 titer and airway responsed to allergen challenge. J Allergy Clin Immunol 92:425-434

23. Bolduc P, Reid L 1976 Mitotic index of the bronchial and alveolar lining of the normal rat lung. Am Rev Respir Dis 114:1121-1128

24. Ayers M, Jeffery PK 1988 Proliferation and differentiation in mammalian airway epithelium. Eur Respir J 1:58-80

25. Evans MJ, Cabral-Anderson LJ, Freeman G 1977 Effects of $\mathrm{NO}_{2}$ on the lungs of aging rats II cell proliferation. Exp Mol Path 27:366-376

26. Busse W, Elias J, Sheppard D, Banks-Schlegel B 1999 NHLBI Workshop summary. Airway remodeling and repair. Am J Respir Crit Care Med 160:1035-1042

27. Bai TR, Cooper J, Koelmeyer T, Pare PD, Weir TD 2000 The effect of age and duration of disease on airway structure in fatal asthma. Am J Respir Crit Care Med $162: 663-669$

28. Hershenson MB, Kelleher MD, Naureckas ET, Abe MK, Rubinstein VJ, Zimmermann A, Bendele AM, McNulty JA, Panettiieri RA, Sloway J 1994 Hyperoxia increased airway cell s-phase traversal in immature rats in vivo. Am J Respir Cell Mol Biol 11:296-303

29. Panettieri Jr R 1998 Cellular and molecular mechanisms regulating airway smooth muscle proliferation and cell adhesion molecules expression. Am J Respir Crit Care Med 158:S133-S140

30. Ebina M, Takahashi T, Chiba T, Motomiya M 1993 Cellular hypertrophy and hyperplasia of airway smooth muscles underlying bronchial asthma. Am Rev Respir Dis 148:720-726 (H-E). In selected animals, the Luna staining was also performed to observe an infiltration of eosinophils in the airways. 